\title{
Design e implementação do whitetopping na restauração de rodovias
}

\author{
RAFAEL ELER DE SOUZA - Mestre em Engenharia de MateriaIS \\ SEBASTIANA LUIZA BRAGANÇA LANA - PhD em Engenharia de Materials \\ GILBERTO HENRIQUE T. ÁLVARES DA SILVA - DoUTOR EM CIÊNCIAS \\ Seleção e Análise de Materials, Rede Temática de Materials - Redemat
}

\section{RESUMO}

AS RODOVIAS BRASILEIRAS SÃO RESPONSÁVEIS POR 96\% DO TRANSPORTE DE PASSAGEIROS, 62\% DO TRANSPORTE DE CARGAS E NECESSTTAM DE PAVIMENTAÇÃO DE ALTA DURABILDADE E BAIXO CUSTO DE MANUTENÇÃO, CAPAZES DE SUPORTAR O TRÁFEGO INTENSO, PESADO E REPEITTIVO. UM DOS MÉTODOS QUE REDUZ OS CUSTOS DA CONSERVAÇÃO DE PAVIMENTOS, POIS ELIMINA TANTO OS CUSTOS DE REMOVER A ANTIGA PAVIMENTAÇÃO ASFÁLTICA QUANTO OS DE CONSTRUIR UMA NOVA BASE, É MUNDIALMENTE CONHECIDO COMO WHITETOPPING, QUE SE VALE DA GRANDE RESIS-
TÊNCIA À COMPRESSÃO E À ABRASÃO DO CONCRETO PARA AMPLAR A SOBREVIDA DAS VIAS, COM A MÍNIMA MANUTENÇÃO.

NUMA EXTENSA REVISÃO BIBLOGRÁFICA DISCUTE-SE OS TIPOS, O DIMENSIONAMENTO E AS PRINCIPAIS INOVAÇÕES DESTE MÉTODO DE PAVIMENTAÇÃO E CONCLUI-SE QUE A MELHOR PERFORMANCE DESTE REVESTIMENTO FOI ALCANÇADA COM A APLICAÇÃO COM PAINÉIS DE LAJES COM 1.83 M X 1.83 M (6 PÉS X 6 PÉS), DE FORMA A DIMINUIR AS TENSÕES DAS RODAS SOBRE A PAVIMENTAÇÃO E O RISCO DA FALHA POR FADIGA.

Palavras-chave: whitetopping, concreto, pavimentação, rodovias, recuperação.

\section{INTRODUÇÃO}

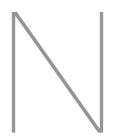

as últimas décadas, o peso dos veículos aumentou drasticamente, resultando na deterioração precoce dos pavimentos. Muitos pavimentos de asfalto atingiram prematuramente o fim de sua vida útil ou apresentaram altos níveis de desgaste [1]. As rodovias brasileiras são responsáveis por $96 \%$ do transporte de passageiros, $62 \%$ do transporte de cargas e necessitam, portanto, de pavimentação de alta durabilidade e baixo custo de manutenção, capazes de suportar o tráfego intenso, pesado e repetitivo [2].

Para suprir essa demanda, as agências rodoviárias têm investido em estratégias de manutenção bem-sucedidas para as redes rodoviárias, apesar dos orçamentos apertados, do aumento do volume de tráfego e das cargas, bem como do foco crítico que surgiu com a sustentabilidade e conservação do pavimento. Em muitas atividades de conservação de pavimentos, o recapeamento com uma camada de concreto colado pode representar uma solução mais econômica, de construção rápida e sustentável, do que a reconstrução completa [1, 3].

A aplicação de uma camada de concreto de cimento Portland diretamente sobre a superfície do pavimento existente é um dos métodos idealizados para reduzir

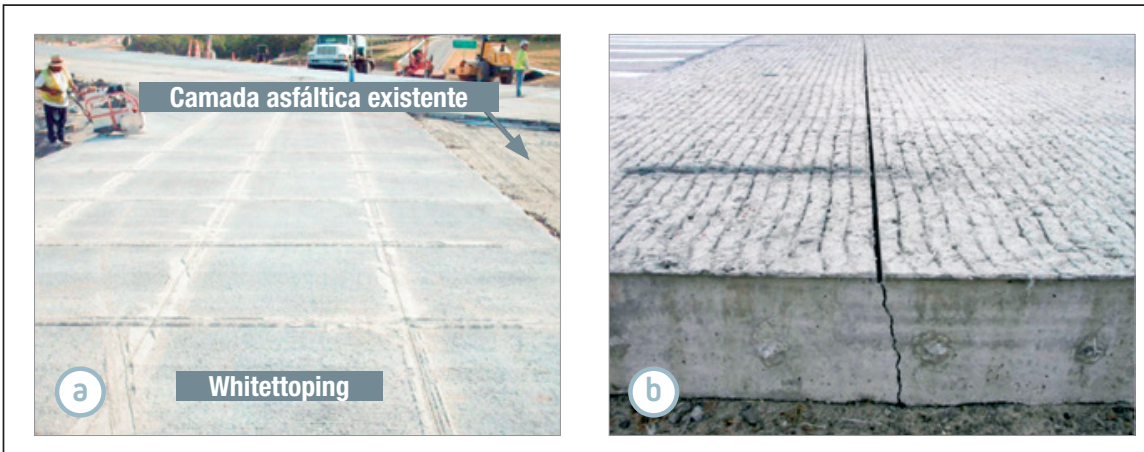

Figura 1

Aplicação da camada de concreto sobre a pavimentação existente: (a) lajes sobre o pavimento; (b) lajes em detalhe Fonte: adaptado de Chen, Won [6] 


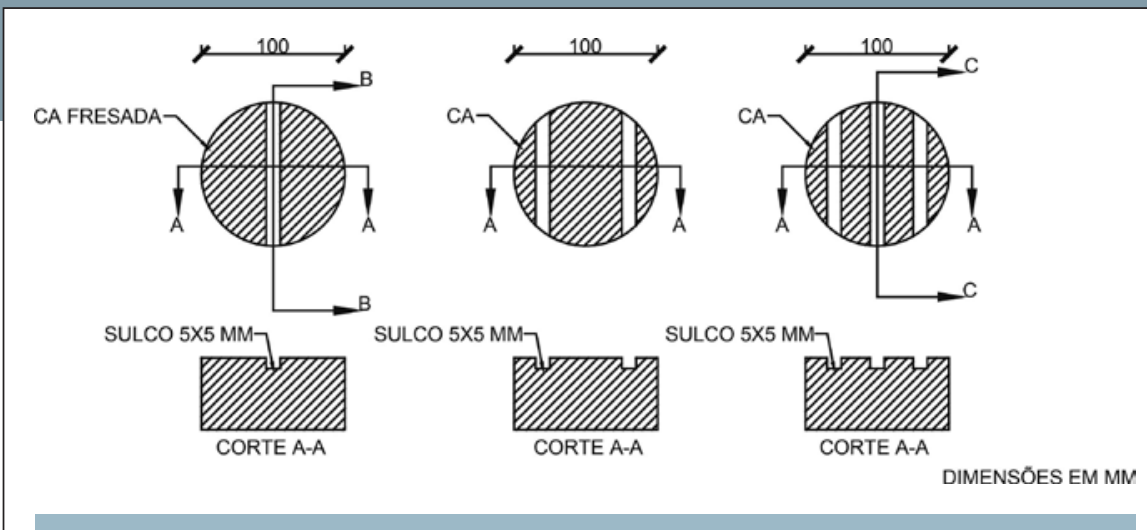

Figura 2

Superfície fresada com ensaios sobre os tipos de sulcos

Fonte: adaptado de Suresha and Satish [5]

os custos da conservação de pavimentos [4]. Este processo elimina tanto os custos de remover a antiga pavimentação asfáltica quanto aqueles de construir uma nova base (Fig. 1). Este método é mundialmente conhecido como whitetopping e vale-se da grande resistência à compressão e à abrasão do concreto, ampliando a sobrevida das vias com a mínima manutenção e permitindo que o composto atue como uma camada monolítica [5].

O whitetopping, incluindo "in lays" (aplicado em locais de sub-leito fraco), foi amplamente usado nos EUA e na Europa entre os anos 40 e 60 para sobrepor pavimentos flexíveis existentes. Várias espessuras de concreto têm sido usadas dependendo das cargas de tráfego esperadas [7]. É utilizado há mais de 25 anos pelo departamento de rodagens de $\mathrm{Ne}-$ brasca (USA) e, com o passar do tempo, em todo mundo [8-10].

Desde os anos 90, o whitetopping ultrafino (WTUF) tem sido amplamente utilizado na reabilitação e reforço de pavimento asfáltico. É registrado que aproximadamente 2.200.000 $\mathrm{m}^{2}$ de estrada com WTUF foram construídos nos Estados Unidos até o final de 2000 [11].

O whitetopping é classificado em três subcategorias com base na espessura: whitetopping convencional (> $200 \mathrm{~mm}$ ), o fino (100-200 mm) e o ultrafino (50-100 mm). O concreto pode ser aderido sobre o asfalto em três formas: como sobreposições coladas diretamente sobre o asfalto, pinçados ou em sulcos (in lays) $[4,12]$. Na preparação da pavimenta- ção existente, o fluxo de trabalho da rodovia e o tipo de compósito a ser empregado na construção é o que determina o tipo de técnica de adesão [13].

Os benefícios do revestimento de concreto colado podem ser alcançados simultaneamente ao mecanismo de colagem. Quando o revestimento e o pavimento existente estiverem bem unidos, este novo pavimento irá comportar-se como uma estrutura monolítica para suportar as tensões do transporte e suas cargas. Portanto, a ligação entre a sobreposição e o pavimento existente é muito importante para evitar problemas iniciais e garantir um bom desempenho [14].

O concreto de alto desempenho com cimento com elevada resistência à compressão (CP V) [15] é, normalmente, o preferido. A alta resistência e bom desempenho do concreto produzido com CP Vé alcançado utilizando cimentos aditivados com microssílica para o endurecimento rápido. Neste contexto, dois terços da resistência final do concreto são atingidos em um período de 48 horas. Finalmente, o pavimento pode ser aberto ao tráfego em até 72 horas após sua implantação. Concretos utilizados nos projetos de whitetopping ultrafino e fino são geralmente fundamentados a partir da relação água/cimento menor ou inferior a 0,40\% [16].

No presente estudo, exploramos a bibliografia em relação ao estado da arte no design da pavimentação e restauração por whitetopping. Os processos foram abordados trazendo à luz os mecanismos de funcionamento do whitetopping, possí- veis problemas na implementação e soluções plausíveis.

\section{DESENVOLVIMENTO}

\section{I Métodos}

No Brasil, não existe uma normalização atribuída ao whitetopping pela Associação Brasileira de Normas Técnicas (ABNT). No entanto, o Departamento Nacional de Infraestrutura de Transportes (DNIT) referendou a norma 068/2004 conferida ao pavimento rígido. Ademais, a norma não contempla as informações sobre os concretos com cargas e fibras, resultando numa menor espessura e maior qualidade de pavimentação.

A partir do banco de dados CAPES, foi feita uma busca utilizando as palavras chave "whitetopping", "fadiga em whitetopping", "design implantation of whitetopping" e "bond strength of whitetopping". Foi analisado noventa artigos e selecionados vinte e três, baseados nos pontos de interesse bem como nas datas de publicação. Foram também pesquisadas as normalizações pertinentes como as da ABNT e do DNIT.

Foram revisados um total de vinte publicações sobre a recuperação da pavimentação através de um método ainda pouco explorado no Brasil.

\subsection{Tipos de Whitetopping}

O whitetopping pode ser classificado em três tipos, com base no grau de ligação entre a camada asfáltica existente (CA) subjacente e a sobreposição do concreto de cimento Portland (CP), bem como a espessura da sobreposição.

Whitetopping convencional (WT): consiste em uma cobertura de $\mathrm{CP}$ com espessura de $200 \mathrm{~mm}$ ou mais (no topo da camada betuminosa existente). É projetada e construída sem levar em consideração qualquer ligação entre a cobertura de concreto e a camada betuminosa subjacente - 
ou seja, com uma interface não ligada. O whitetopping convencional é projetado e construído como um novo pavimento rígido, sem assumir nenhuma ação composta. Ele trata a superfície betuminosa já existente como uma sub-base de concreto enxuto seco, sendo que a condição da superfície betuminosa existente não importa significativamente, exceto quando a superfície betuminosa possui danos isolados como subsidência $[5,16,17]$.

- Whitetopping fino (WTF): quando o CP tem espessura maior que 100 mm e menor que $200 \mathrm{~mm}$. Apesar de não ser obrigatória, a ligação entre o CP sobreposto e a camada betuminosa subjacente é muitas vezes realizada frequentemente com interface parcialmente ligada e com concreto de alta resistência. Quando a ligação entre as camadas no projeto for ignorada, o concreto de alta resistência com fibras é comumente usado. As juntas têm espaçamentos menores de 0,6 a $1,25 \mathrm{~m}[5,16,17]$.

- Whitetopping ultrafino (WTUF): quando a camada de CP com interface colada possui espessura igual ou inferior a 100 mm. A sobreposição e fixação da camada de CP sobre uma camada betuminosa subjacente é obrigatória. A ligação entre a superfície betuminosa e a cobertura de CP é estabelecida através de uma fresagem com profundidade média de $25 \mathrm{~mm}$. Uma ligação eficaz é fundamental para um melhor desempenho do WTUF. Essa ligação pode ser fornecida por alguns outros métodos, mas a fresagem é considerada desejável porque a ligação eficaz entre a superfície existente e a sobreposta é absolutamente essencial [5, 16, 17].

\subsection{Concreto com fibras no whitetopping}

O uso de pequenas fibras no WTUF tem se tornado mais popular na

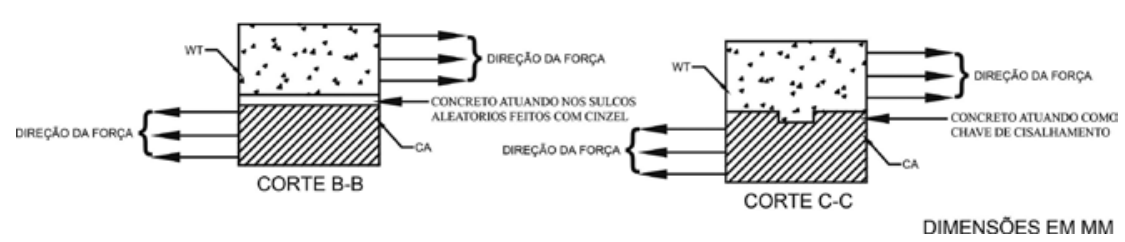

Figura 3

Aplicação do WT sobre a pavimentação e o funcionamento das chaves de cisalhamento: (b-b) concreto sobre sulcos aleatórios; (c-c) concreto atuando como chave de cisalhamento

Fonte: adaptado de Suresha and Satish [5]

restauração de pavimentos. A distribuição aleatória de fibras dentro da matriz de concreto compartilha tensões através das fissuras iniciais [1]. Além de avaliar o efeito do uso de diferentes tipos e conteúdo das fibras, o WTUF pode também ser usado para comparar diferentes graus de aderência na interface substrato-sobreposição. A resistência da interface pode ser deteriorada devido às diferentes rugosidades (escarificação) ou devido à falta de limpeza do substrato (uma situação de campo comum) [4].

O concreto de alta resistência com fibras é normalmente feito com juntas pouco espaçadas (no intervalo de 0,6 a $1,25 \mathrm{~m})$. O WTUF de $50 \mathrm{~mm}$ a $75 \mathrm{~mm}$ geralmente não é recomendado quando há gradientes de temperaturas altas e em locais com grande fluxo de tráfego.

Além disso, métodos de aplicação desta técnica podem estar indisponíveis dependendo da localidade. No entanto, para estradas internas de municípios menores, onde o tráfego é restrito e controlado, a aplicação de uma fina camada de whitetopping com fibras pode ser considerada [16].

\subsection{Dimensionamento do whitetopping}

O projeto e a construção de WT e WTF são iguais a um novo pavimento rígido, sem assumir ação composta alguma. No caso da WTUF, a ligação entre a cobertura de concreto e a camada de asfalto subjacente é fundamental para sustentar o período de serviço desejado [5]. Podem ser construídos com lajes de grande porte de 3,05 $\mathrm{m} \times 3,66 \mathrm{~m}$ (10 pés $\times 12$ pés), médio porte, como 1,83 $\mathrm{m} \times$ $1,83 \mathrm{~m}$ (6 pés $\times 6$ pés) e 1,83 $\mathrm{m} \times 1,52 \mathrm{~m}$ ( 5 pés $\times 6$ pés) e lajes menores, com tamanho menor que $1,37 \mathrm{~m} \times 1,37 \mathrm{~m}(4,5$ pés $\times 4,5$ pés) [15].

De acordo com Suresha and Satish [5], pesquisas dos anos 90 usaram técnicas como vassoura mecânica, vassoura mecânica com jato de ar, moagem, cimento e rejuntes de baixa densidade e revestimento de aderência por emulsão, para melhorar a ligação entre o concreto e a base asfáltica. Nos estudos, os autores notaram que a seção do pavimento de whitetopping com aderência e de resistência tão baixa quanto 0,690 MPa não apresentou desgaste.

Portanto, eles sugeriram que a superfície asfáltica existente deve ser fresada e limpa antes da colocação do WTUF (Fig. 2). Além do mais, foi enfatizado a necessidade de investigar o papel da resistência ao cisalhamento da interface em relação a possíveis falhas. Um recorte circular na superfície do CA atua como uma chave de cisalhamento (Fig. 3). Isso pode ser criado colocando o colarinho de cisalhamento de dimensão necessária no centro da placa de base do molde Marshall e, subsequentemente, compactando a mistura CA solta (figura 4 a).

Segundo DNER-ME 043/95 [18], o molde Marshall é um molde de compactação de aço, consistido de anéis superior e inferior e de uma placa base. A placa base e o anel superior devem encaixar-se perfeitamente nas extremidades do anel inferior e é utilizada principalmente parar determinar a estabilidade e a fluência de 


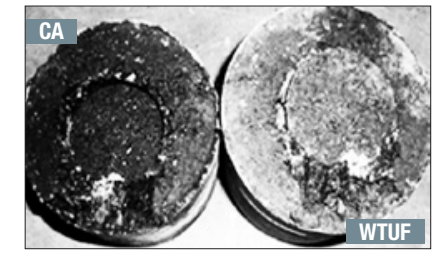
de cisalhamento
(כ) Interface com chave

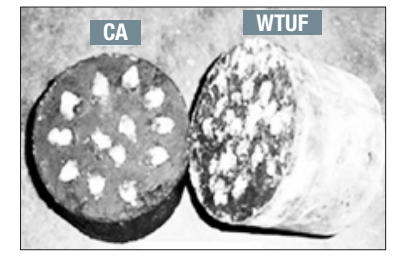

(b) Superfície cinzelada
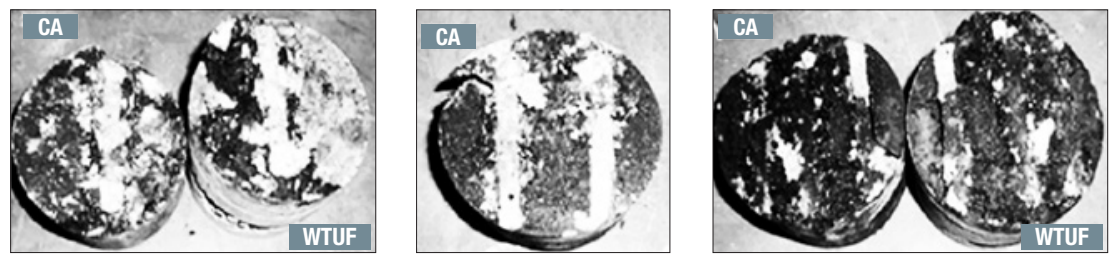

Superfície com sulcos espaçados

\section{Figura 4}

Ensaios sobre os tratamentos superficiais para atestar a ligação entre CA e o WTUF: (a), Colarinho funcionando como chave de cisalhamento;

(b), Superfície cinzelada; (c) Superfície com sulcos espaçados

Fonte: adaptado de Suresha and Satish [5]

misturas betuminosas de asfalto que são utilizados na pavimentação de rodovias, estradas e ruas, e podem ser adotados diversos tipos de tratamentos superficiais.

Suresha and Satish [5] compararam alguns tipos de tratamentos e suas respectivas resistências ao cisalhamento. Os tratamentos de interface foram comparados com as de amostras do compósito WTUF-CA com tratamento de superfície plana, no qual o WTUF foi colocado diretamente no asfalto. No primeiro tipo de tratamento de interface (Fig. 4 b), a superfície asfáltica foi cinzelada aleatoriamente. No segundo tipo de tratamento de interface (Fig. 4 c), os corpos de prova compostos foram fornecidos com sulcos. Suas conclusões mostraram que compostos WTUF-CA, sem o tratamento de interface adequado, podem não garantir a ligação entre WTUF e CA e o melhor tratamento de interface para pavimentos WTUF-CA seria sulcos com espaçamento adequado [5].

Segundo o Indian Roads Congress [16], a pavimentação por whitetopping deve atingir uma resistência à compressão mínima de $40 \mathrm{~N} / \mathrm{mm}^{2}$ e uma resistência à flexão mínima de 5,0 N/mm². A resistência ou módulo de ruptura (carregamento do terceiro ponto) do concreto deve ser 4,5 MPa, correspondendo ao grau mínimo do concreto com a resistência à compressão de $40 \mathrm{~N} / \mathrm{mm}^{2}$ aos 28 dias. No entanto, é preferível ter uma resistência à flexão de 5,0-6,0 MPa. Para isso, as misturas devem ser projetadas de acordo com IRC: 44 [19] ou IS: 10262[20].

A análise via Método dos Elementos Finitos (MEF), conduzida para comparar a tensão na parte inferior da sobreposição para diferentes condições de aderência, demonstrou a importância da ligação das camadas. A Fig. 5 mostra a importância da ligação de interface. Quando a cobertura de concreto e a camada de asfalto são aderidas (Fig. 5 a), a tensão induzida por carga na parte inferior da camada de concreto é menor do que quando as camadas não estão aderidas, como visto na Fig. 5 (b). [17].

Todos os métodos atuais de projeto de WTUF descrevem a razão de tensões totais aplicadas sobre a capacidade do pavimento e dependem do conceito de razão de tensões para determinar a espessura do WTUF. A capacidade do pavimento é calculada a partir da resistência à flexão (ou seja, módulo de ruptura, MOR) de vigas de concreto representativas, que é determinada pela fórmula elástica (Eq. (1) da ASTM C78. O valor de MOR apresenta o modulo de ruptura do concreto até o aparecimento de fissuras na matriz de concreto. As fibras quando presentes, desempenham um papel significativo e eficaz dentro da matriz do concreto após o aparecimento da fissura inicial durante a fase inelástica [1].

MOR $=\frac{P_{\max } \times L}{b x d^{2}}$

Onde:

MOR: módulo elástico para ruptura, MPa ou psi;

$\mathrm{P}_{\text {max }}$ : carga gravada na primeira quebra, N ou lb;

L: vão da vida, mm ou in;

B: largura da viga, $\mathrm{mm}$ ou in;

D: altura da viga, $\mathrm{mm}$ ou in.

Os quatro fatores de maior influência na implantação do whitetopping são: temperatura, idade, tratamento das camadas e tamanho da laje do pavimento [3-6, 11, 17, 21-23].

Independente da espessura da laje, o layout do painel deve ser de 1,83 m $\times 1,83 \mathrm{~m}$ ( 6 pés $\times 6$ pés). Ao afastar as juntas serradas dos caminhos das rodas, as tensões de carga da roda nas lajes de concreto são reduzidas. Ademais, o aumento das tensões de ondulação devido ao uso deste tamanho de laje em comparação com outras menores parece ser compensado pela redução nas tensões da carga. A espessura não deve ser inferior a $7 \mathrm{~cm}$, sendo recomendado o asfalto com módulo de elasticidade acima de $1.500 \mathrm{MPa}$ como matéria-prima para a cobertura asfáltica. Por fim, a laje WTUF deverá ser limitado entre 1,8 e 1,2 m, enquanto sua espessura de ser mantida entre 7 a $10 \mathrm{~cm}$ [11]. 


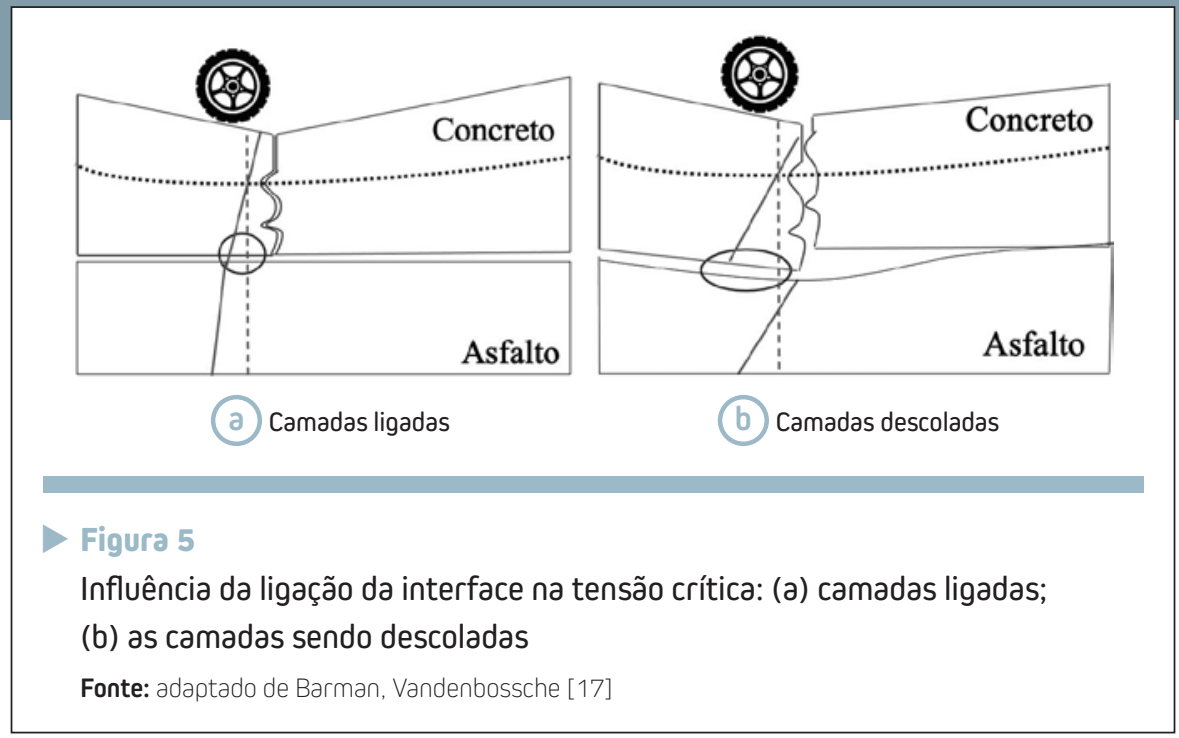

Além do tamanho de laje, matéria prima e juntas já mencionados, a qualidade da base e sua preparação deve ser considerada de forma a adquirir maior atrito e estabilidade entre a base e o whitetopping, criando uma estrutura monolítica, sem vazios para não haver acumulo de tensões e possíveis danos prematuros à estrutura.

\section{CONCLUSÕES}

Foram expostas as principais técnicas de whitetopping que podem reduzir remoção e reconstrução de uma nova pavimentação. Tais métodos já são utilizados países desenvolvidos e podem resultar numa sobrevida da pavimentação superior a 20 anos, diminuindo a periodicidade da manutenção e se tornando economicamente viável.
No Brasil, mesmo nossa malha viária corresponder a quase 100\% do transporte de passageiros e mais de $50 \%$ de carga, a normalização do DNIT não aborda técnicas atuais de whitetopping, sendo este um método eficiente e rápido na reparação de rodovias.

Problemas no design da preparação da base devem ser minimizado para a criação de uma estrutura monolítica, não criando acúmulos de tensões. Outros fatores, como recalque do solo, capilaridade e possíveis dados sobre os problemas que lavaram fratura da cobertura asfáltica, terão influência no método de WT escolhido, visto que as propriedades físicas da cobertura asfáltica e o concreto são distintas.

Dados mais recentes da literatura indicam que:
Existem 3 tipos de whitetopping que podem ser adotados de acordo com o grau da pavimentação existente;

- A largura dos painéis de lajes deve ser entre 1,2 e 1,8 m [6, 11];

- Ensaios com WTUF utilizaram espessuras de lajes entre 7 e $10 \mathrm{~cm}$ [11];

- OWT deve atingir uma resistência à compressão mínima de 40 N/mm² e uma resistência à flexão mínima de $5,0 \mathrm{~N} / \mathrm{mm}^{2}$ [16];

O WTUF feito com concreto de alta resistência e fibras é normalmente construído com juntas pouco espaçadas (no intervalo de 0,6 a 1,25 m) $[5,16,17]$;

- Compostos WTUF-CA, sem o tratamento de interface adequado, podem não garantir a ligação entre WTUF e CA [11];

- O melhor tratamento de interface para pavimentos WTUF-CA seria com sulcos com espaçamento adequado [12];

- O whitetopping ultrafino de $50 \mathrm{~mm}$ a 75 mm geralmente não é recomendado para gradientes de temperaturas altas e locais com alto tráfego [16].

\section{AGRADECIMENTOS}

A agência de fomento CAPES e a REDEMAT.

\section{REFERÊNCIAS BIBLIOGRÁFICAS}

[1] Suresha, S. and D. Satish, Interface Bond Strength of Ultra-Thin Whitetopping (UTW) and Hot Mix Asphalt (HMA) Composites by Direct Shear. Journal of Testing and Evaluation, 2017. 45(6): p. 1999-2008

[2] Chen, D.H., et al., Design improvements to enhance the performance of thin and ultra-thin concrete overlays in Texas. Construction and Building Materials, 2016. 116: p. 1-14.

[3] IRC. Guidelines for Conventional and Thin Whitetopping. in Indian Roads Congress (IRC). 2015. New Delhi.

[4] Lin, D.-F. and H.-Y. Wang, Forensic Investigation of Ultra-Thin Whitetopping Failures in Taiwan. Journal of Performance of Constructed Facilities, 2005. 19.

[5] TÉCNICAS, A.B.D.N., Cimento Portland — Requisitos, 2018. p. 12.

[6] IRC:SP-76, Tentative Guidelines for Conventional, Thin and Ultra-Thin Whitetopping, in Indian Roads Congress2015: New Delhi, India.

[7] Barman, M., J.M. Vandenbossche, and Z. Li, Influence of Interface Bond on the Performance of Bonded Concrete Overlays on Asphalt Pavements. Journal of Transportation Engineering, Part B: Pavements, 2017. 143(3): p. 04017008.

[8] Rodagens, D.N.d.E.d., Misturas Betuminosas a Quente, in Ensaio Marshall1995. p. 11.

[9] Congress, I.R., Guidelines for Cement Concrete Mix Design for Pavements, in Third Revision2017, India Offset Press: Indian Roads Congress

[10] Committee, C.a.C.S., Concrete Mix Proportioning in Guidelines2009, Bureau of Indian Standards: New Delhi, India.

[11] Transportes, D.N.d.I.-E.d., Manual de pavimentos rígidos. 2.ed, in 714, IPR, Editor 2005: Rio de Janeiro. 